\title{
Short-term effect analysis of radiofrequency ablation combined chemotherapy on middle and late period non-small cell lung cancer
}

\author{
SHUO YU, ZHEN ZHOU WU, HONG TAO SI, SAI YANG, GUANG MAO LIU and XIAO DONG ZHAO \\ Department of Oncological Surgery, The Second Hospital of Hebei Medical University, \\ Shijiazhuang, Hebei 050000, P.R. China
}

Received March 18, 2016; Accepted September 1, 2016

DOI: $10.3892 / \mathrm{ol} .2016 .5276$

\begin{abstract}
In this study, we investigated the effect of radiofrequency ablation (RFA) combined chemotherapy on middle and late period non-small cell lung cancer (NSCLC). In total, 85 cases of middle and late period NSCLC patients were selected, and were randomly divided into the RFA combined chemotherapy group, RFA treatment group and chemotherapy group. After treatment, the three groups were followed up for computed tomography (CT) scan, and were analyzed for the effect of RFA combined chemotherapy on middle and late period NSCLC. It was found that CT value of RFA combined chemotherapy group decreased significantly compared to before surgery $(\mathrm{P}<0.05)$. The $\mathrm{CT}$ value of the RFA group decreased significantly compared to before surgery $(\mathrm{P}<0.05)$. The $\mathrm{CT}$ value of chemotherapy group was not significantly changed compared to before surgery ( $\mathrm{P}>0.05)$. The postoperative CT value of the RFA combined chemotherapy group and RFA group was smaller compared to that of the chemotherapy group $(\mathrm{P}<0.05)$. Effective rate [complete response (CR) + partial response (PR)] of RFA combined chemotherapy group was significantly higher than that of the EFA and chemotherapy groups $(\mathrm{P}<0.05)$. Effective rate $(\mathrm{CR}+\mathrm{PR})$ of the RFA group was significantly higher than that of the chemotherapy group $(\mathrm{P}<0.05)$. By contrast, the progressive rate $(\mathrm{P})$ of RFA combined chemotherapy group was significantly lower than that of the RFA and chemotherapy groups $(\mathrm{P}<0.05)$. In conclusion, RFA combined chemotherapy has obvious effect on middle and late period NSCLC, and is safe and feasible.
\end{abstract}

\footnotetext{
Correspondence to: Dr Xiao Dong Zhao, Department of Oncological Surgery, The Second Hospital of Hebei Medical University, 215 Peace West Road, Shijiazhuang, Hebei 050000, P.R. China

E-mail: uxbxemcwmya39@163.com
}

Key words: lung cancer, radiofrequency ablation, chemotherapy

\section{Introduction}

Lung cancer severely compromises human health, and is nowadays one of the most common malignant tumors worldwide (1). Since the prophase of clinical symptoms are not obvious, approximately $80 \%$ of patients are already in middle or late periods while visiting doctors, and thus surgery does not constitute a viable option (2).

In recent years, RFA has been gradually introduced for lung cancer treatment (3). It has key advantages, e.g., precise treatment effect, high safety and small trauma, which has become an important part in non-surgery treatment of lung cancer $(3,4)$. Radiofrequency ablation (RFA) can improve the temperature of tumor tissue in a short period of time and make tumor cells degenerated and necrotic, thus reaching the aim of killing tumor tissue, which has achieved great clinical effect (5-7). Chemotherapy also plays an important role in cancer treatment process. Non-small cell lung cancer (NSCLC) accounts for $80 \%$ of lung cancer types.

In this study, we investigated the experience of middle and late period NSCLC treatment by RFA combined whole-body chemotherapy, single RFA treatment and whole-body chemotherapy treatment. We analyzed the effect of RFA combined chemotherapy on middle and late period NSCLC.

\section{Patients and methods}

General information. A total of 85 cases of NSCLC patients admitted to the Department of Oncological Surgery (Hebei, China) from June, 2013 to December, 2013 were selected. The patients were in clinical phase III or IV, and diagnosed as NSCLC by pathology. There were 50 males and 35 females, aged 44-76 years with a median age of 58 years. There were 35 cases in the RFA combined chemotherapy group, including 22 males and 1 female aged $44-65$ years; 28 cases in the RFA group with 18 males and 10 females aged 58-76 years; and 22 cases in the chemotherapy group with 15 males and 7 females aged 52-72 years.

\section{Treatment methods}

RFA-combined chemotherapy group. The 85 cases were examined for routine blood tests, electrocardiogram and chest 

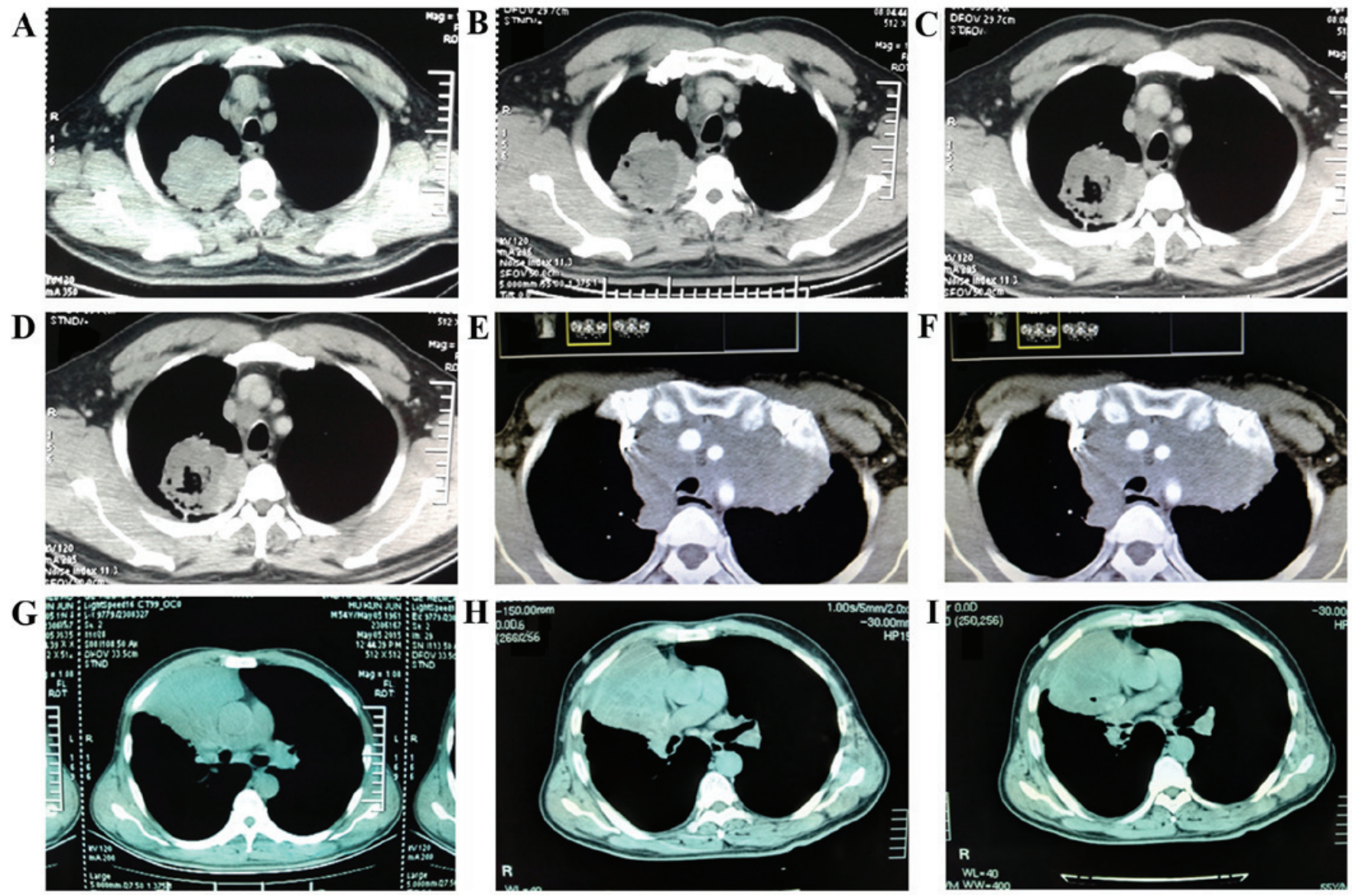

Figure 1. (A-I) Representative CT image showing changes in values for patients in different groups at the indicated time points. CT, computed tomography.

computed tomography (CT) scan before surgery. The RFA treatment under the guidance of CT scan. Cool-tip RFA system (Covidien; Medtronic, Minneapolis, MN, USA) was used, and RFA needle with 20 or $30 \mathrm{~mm}$ was selected according to the size of the tumor. The CT scanning was carried out after localizing marker on body surface, and determining needle insertion point according to body surface localization after selecting treatment layer, and needle insertion angle and depth was measured. Regular sterilization and sheet paving was performed. For anesthesia, 2\% lidocaine hydrochloride was used. The needle was gradually inserted according to needle insertion point and angle, and CT scan was performed to guide needle depth.

The treatment was started when the RFA needle was within the tumor tissue. The treatment power was $120 \mathrm{~W}$, and the time was $10 \mathrm{~min}$. Multiple times of RFA treatment were carried out according to the size of the tumor for complete cover of the tumor tissue according to treatment. After the treatment, necessary care was provided, e.g., oxygen uptake, hemostasis, anti-infection and fluid replacement. After surgery, blood tests were performed again. In the groups without chemotherapy, cisplatin + docetaxel was administered. For chemotherapy, $75 \mathrm{mg} / \mathrm{m}^{2}$ of docetaxel was added to $0.9 \% \mathrm{NaCl}$ of $250 \mathrm{ml}$, intravenous drip on the first day. Along with $75 \mathrm{mg} / \mathrm{m}^{2}$ of docetaxel $500 \mathrm{ml}$ of $0.9 \% \mathrm{NaCl}$ was added for 3 days, separately. The chemotherapy plan included 6 treatment courses with 21 days of a treatment course.
RFA group. Treatment methods were the same as the radiofrequency methods in the RFA combined chemotherapy group.

Chemotherapy group. The treatment methods and period was the same as the chemotherapy plan in the RFA combined chemotherapy group.

Patients in the three groups were re-examined for a CT scan 3 and 6 months after surgery. During this process, treatment was provided on time if there was bleeding in local tumor tissue in RFA combined chemotherapy group and RFA group.

\section{Data collection and statistical processing}

CT value and tumor sizes. For all groups, the tumor CT scan and tumor size (longest diameter) was measured at each follow-up. SPSS 13.0 statistical software for Windows (IBM; Chicago, IL, USA) was used to analyze CT value. The results were expressed as mean $\pm \mathrm{SD}$, and the Student's t-test was used to carry out statistical analysis. $\mathrm{P}<0.05$ was considered to indicate a statistically significant difference.

The effects were assessed 6 months after RFA surgery. SPSS 13.0 statistical software was used to assess treatment effect. The results are expressed as percentage (\%), and $\chi^{2}$ test used for statistical analysis, with $\mathrm{P}<0.05$ considered to indicate a statistically significant difference.

\section{Results}

Change of CT value. In the RFA combined chemotherapy group, the CT value before surgery was $48.9 \pm 12.7 \mathrm{HU}$ (Fig. 1A), 
Table I. Changes of CT value.

\begin{tabular}{lccc}
\hline Groups & $\begin{array}{c}\text { CT value } \\
\text { before } \\
\text { surgery (HU) }\end{array}$ & $\begin{array}{c}\text { CT value } \\
\text { 3 months after } \\
\text { surgery (HU) }\end{array}$ & $\begin{array}{c}\text { CT value } \\
6 \text { months after } \\
\text { surgery (HU) }\end{array}$ \\
\hline $\begin{array}{l}\text { RFA combined } \\
\text { chemotherapy }\end{array}$ & $48.9 \pm 12.7$ & $27.5 \pm 10.8$ & $24.6 \pm 11.2$ \\
$\begin{array}{l}\text { RFA } \\
\text { Chemotherapy }\end{array}$ & $50.4 \pm 13.5$ & $30.4 \pm 11.0$ & $26.6 \pm 14.7$ \\
\hline
\end{tabular}

CT, computed tomography; RFA, radiofrequency ablation.

Table II. Effect assessment.

\begin{tabular}{lcrrr}
\hline Groups (n) & CR (n) & PR, n (\%) & SD, n (\%) & P, n (\%) \\
\hline $\begin{array}{l}\text { RFA combined } \\
\text { chemotherapy (35) }\end{array}$ & 0 & $28(80.0)$ & $5(14.3)$ & $2(5.7)$ \\
$\begin{array}{l}\text { RFA (28) } \\
\text { Chemotherapy (22) }\end{array}$ & 0 & $18(64.3)$ & $5(17.9)$ & $5(17.9)$ \\
& 0 & $6(27.3)$ & $10(45.5)$ & $6(27.3)$
\end{tabular}

CR: clinical symptoms completely disappear, lumps completely regress; PR: clinical symptoms improved, product of multiplication of tumor longest diameters decreased by $>50 \%$; SD: lump decrease is $<50 \%$ or without obvious change and maintain $>6$ months; $\mathrm{P}$ : clinical symptoms aggravated, lump enlarged. $\mathrm{CR}$, complete response; PR, partial response; $\mathrm{SD}$, stable disease.

3 months after surgery was $27.5 \pm 10.8 \mathrm{HU}$ (Fig. 1B), and 6 months after surgery $24.6 \pm 11.2 \mathrm{HU}$ (Fig. 1C). In RFA group, CT value before surgery was $50.4 \pm 13.5 \mathrm{HU}$ (Fig. 1D), 3 months after surgery it was $30.4 \pm 11.0 \mathrm{HU}$ (Fig. 1E), and 6 months after surgery $26.6 \pm 14.7 \mathrm{HU}$ (Fig. 1F). For the chemotherapy group, the CT value before surgery was $45.4 \pm 15.0 \mathrm{HU}$ (Fig. 1G), 3 months after surgery it was $43.1 \pm 15.8 \mathrm{HU}$ (Fig. $1 \mathrm{H}$ ), and 6 months after surgery $46.2 \pm 13.5 \mathrm{HU}$ (Fig. 1H) (Table I).

Before surgery, CT value of the three groups were not statistically significant $(\mathrm{P}>0.05)$. CT value of RFA combined chemotherapy group decreased significantly after surgery $(\mathrm{P}<0.05)$. CT value of RFA group decreased significantly after surgery $(\mathrm{P}<0.05)$. CT value of chemotherapy group did not change significantly $(\mathrm{P}>0.05)$. Compared with chemotherapy group, CT value of RFA combined chemotherapy group and RFA group decreased significantly after surgery $(\mathrm{P}<0.05)$ (Table II).

The effect assessment. In RFA combined chemotherapy group, there was no complete response (CR) (0/35), partial response (PR) 80\% (28/35), stable disease (SD) 14.3\% (5/35), P 5.7\% (2/35). In the RFA group there was no CR (0/28), PR $64.3 \%$ (18/28), SD $17.9 \%$ (5/28), P 17.9\%(5/28). In the chemotherapy group, there was no CR (0/22), PR $27.3 \%$ (6/22), SD $45.5 \%$ (10/22), P $27.3 \%$ (6/22) (Table II).

The effective rate $(\mathrm{CR}+\mathrm{PR})$ of the RFA combined chemotherapy group was higher than that of the RFA and chemotherapy groups $(\mathrm{P}<0.05)$. By contrast, the progressive rate $(\mathrm{P})$ of the RFA combined chemotherapy group was significantly lower than that of the RFA and chemotherapy groups $(\mathrm{P}<0.05)$ (Table II).

Postoperative complications. In the RFA combined chemotherapy group, there was no death related to operation; 3 cases of chest pain, 5 cases of pneumothorax, 1 case received cavitas thoracis paracentesis once, 1 case needed cavitas thoracis closed type drainage, whereas in other 3 cases cavitas thoracis was absorbed spontaneously; 7 cases have sputum with blood, no hemoptysis, no intra-lung bleeding; 15 cases had fever, with the highest temperatures under $38.5^{\circ} \mathrm{C}$, and were obviously improved after physical cooling. For the RFA group, there was no death related to operation; 3 cases chest pain, 4 cases of pneumothorax, 1 case received cavitas thoracis paracentesis 1 time in and after surgery respectively, and other 3 cases absorbed by themselves; 5 cases had sputum with blood, 1 cases hemoptysis, no intra-lung bleeding; 12 cases had fever, with the highest temperatures under $38.5^{\circ} \mathrm{C}$, and obvious improvement after physical cooling.

\section{Discussion}

In recent years, there has been a gradual increase of lung cancer incidence (1), which is now the malignant tumor with the highest incidence and mortality. There is a study indicating that a 5-year survival rate of phase I NSCLC patients without treatment is only $6 \%$, and the median survival time is 9-14 months (2). According to statistics, $<20 \%$ patients can be offered surgery. Therefore, the position of non-surgery in lung cancer treatment becomes more important.

RFA is a new technique aiming at tumor treatment. The principle uses high frequency electromagnetic waves produced by radiofrequency electric current to make intra-cellular polar molecules agitate and friction to generate heat, leading to protein degeneration and leading to irreversible clotting necrosis, and thus to killing of tumor cells. RFA has already achieved good effect in liver cancer treatment. Since there is ample blood circulation and breathing heat elimination in the lung, the heat can dissipate, it has been shown that the amount of blood circulation is low in lung tumor tissue, while a high amount of air in lung can speed the accumulation of heat, which causes rapid temperature increase, known as 'side-effect', therefore, RFA has obvious advantage when applied to lung cancer treatment $(3,4)$. RFA can kill the cancer cells in treatment area, reduce tumor load, as well as increase the sensitivity of cancer cells to chemotherapy medicine, which is an important component of the comprehensive treatment to middle and late period NSCLC patients (5). Compared to traditional surgery treatment, RFA has obvious advantages including precise treatment effect, small lesion, short surgery time, fast recovery, capacity of repeated treatment, less complications and high safety level $(6,7)$. The main complications are pneumothorax, fever, thoracalgia, cough and hemoptysis. Most complications have slight symptoms and only a few need special treatment.

Chemotherapy for NSCLC is currently mainly based on platinum, which greatly improves the survival time and quality of life of the patients (8). There is a study comparing the clinical effect of three generations of anticancer drugs. 
Docetaxel and cisplatin or TP plan has become the first-tier chemotherapy plan for NSCLC (8).

In the present study, RFA accomplished precise localization under the guidance of CT, and we designed a needle spread plan according to CT image and treatment range of RFA, making the radiofrequency treatment range fully cover the tumor tissue. It can treat multiple tumor lesions at one time or treat the same lesion many times, as there is no blood supply at the tumor tissue clotting necrosis area after RFA treatment (9). However, the tumor size may not change or even increases, considering it may be related to tumor tissue clotting necrosis, oedema or surrounding acute inflammation (10). Therefore, we can assess the treatment effect according to the results of postoperative-intensified $\mathrm{CT}$, and take multiple RFA according to the results to kill the remaining tumor tissue or completely block the blood supply of tumor tissue, to achieve better treatment effect. The research findings have shown that the lesion may increase 1-3 months after RFA, and gradually decrease after months (11). If there is not obvious decrease of the tumor volume but intensified CT examination shows no change of the CT value, then it indicates the treatment is effective (11). This study showed that in some patients, follow-up CT scan revealed no obvious tumor volume changes in short-term after RFA or a small portion of cases had their tumor volume increased, and all of the CT scan values significantly decreased compared to before, and some cases it showed obvious cavity and diffluented necrosis in the tumor tissue. It suggested the effectiveness of the treatment, which was consistent with research results by different group (12). The CT value of the RFA combined chemotherapy group significantly decreased, while its effective rate $(\mathrm{CR}+\mathrm{PR})$ was higher than that of the RFA and chemotherapy groups, indicating effective treatment.

The findings of the present study show that RFA and chemotherapy have an important function in the treatment of middle and late period NSCLC. In the treatment of the middle and late period NSCLC, RFA combined chemotherapy can improve the treatment effect, and retard pathogenetic progress, with convenient operation, small side-effects and can be repeatedly applied, which is worthy of generalization. Some studies indicated that although compared to traditional chemoradiotherapy, single RFA treatment for lung cancer has the advantage of being able to control local lesion and improving life quality of patients, although it cannot improve survival rate of patients with local late period NSCLC $(13,14)$. However, this study only investigated the short-term effect of RFA-combined chemotherapy on the middle and late period NSCLC. Further studies are required to investigate whether it can improve the long-term effect of factors such as tumor recurrence and metastasis, survival period and quality of life.

\section{References}

1. Ferlay J, Shin HR, Bray F, Forman D, Mathers C and Parkin DM: Estimates of worldwide burden of cancer in 2008: GLOBOCAN 2008. Int J Cancer 127: 2893-2917, 2010.

2. Raz DJ, Zell JA, Ou SH, Gandara DR, Anton-Culver H and Jablons DM: Natural history of stage I non-small cell lung cancer: implications for early detection. Chest 132: 193-199, 2007.

3. Liu B, Liu L, Li Y, Wang H, Hu M, Qian K, Wang R and Zhi X: Survival after radiofrequency ablation for 100 cases of lung neoplasms. Zhongguo Fei Ai Za Zhi 14: 335-339, 2011 (In Chinese).

4. Oshima F, Yamakado K, Akeboshi M, Takaki H, Nakatsuka A, Makita $\mathrm{M}$ and Takeda K: Lung radiofrequency ablation with and without bronchial occlusion: experimental study in porcine lungs. J Vasc Interv Radiol 15: 1451-1456, 2004.

5. Zhao J, Wu Y, Wang Y, et al: Treatment of local late period NSCLC by RFA combined chemoradiotherapy. Cancer Res Prev Treat 8: 495-497, 2004.

6. Iguchi T, Hiraki T, Gobara H, Mimura H, Fujiwara H, Tajiri N, Sakurai J, Yasui K, Date H and Kanazawa S: Percutaneous radiofrequency ablation of lung tumors close to the heart or aorta: evaluation of safety and effectiveness. J Vasc Interv Radiol 18: 733-740, 2007.

7. Qian K, Zhang Y, Zhi XY, Liu BD, Su L, Li Y and Wang H: Study on the safety of radiofrequency ablation in patients with lung cancer above 70 years old. Chinese J Clinicians (Electronic Edition) 7: 5332-5334, 2013 (In Chinese).

8. Grossi F, Aita M, Defferrari C, Rosetti F, Brianti A, Fasola G, Vinante O, Pronzato P and Pappagallo G: Impact of thirdgeneration drugs on the activity of first-line chemotherapy in advanced non-small cell lung cancer: a meta-analytical approach. Oncologist 14: 497-510, 2009.

9. Goldberg SN, Gazelle GS and Mueller PR: Thermal ablation therapy for focal malignancy: a unified approach to underlying principles, techniques, and diagnostic imaging guidance. AJR Am J Roentgenol 174: 323-331, 2000.

10. Liu B, Zhi X, Liu L, Hu M, Wang R, Xu Q, Zhang Y and Su L: Evaluation of three-dimensional reconstruction $\mathrm{CT}$ in percutaneous radiofrequency ablation (RFA) of the unresectable lung tumor with a clustered electrode. Zhongguo Fei Ai Za Zhi 12: 775-779, 2009 (In Chinese).

11. Anderson EM, Lees WR and Gillams AR: Early indicators of treatment success after percutaneous radiofrequency of pulmonary tumors. Cardiovasc Intervent Radiol 32: 478-483, 2009.

12. Higaki F, Okumura Y, Sato S, Hiraki T, Gobara H, Mimura H, Akaki S, Tsuda T and Kanazawa S: Preliminary retrospective investigation of FDG-PET/CT timing in follow-up of ablated lung tumor. Ann Nucl Med 22: 157-163, 2008.

13. Wang J, Wang Y and Zhao J: Short-term and long-term effect of RFA on local late period NSCLC. Int Med Health Guidance News 12: 20-23, 2006 (In Chinese).

14. Mohamed Lotayef, Azza Taher, Hanna Attia,Azza Nasr, Hisham El Hossieny, Mohammed Mahmoud and Noha Essam: A clinic-epidemilogical study of cases of locally advanced non small cell lung cancer (NSCLC) that received radiotherapy at NCI Cairo in the period from 2001-2010. J Cancer Ther 5: 542-551, 2014. 